\title{
RECUPERACIÓN DEL FRENTE MARÍTIMO DE EL CAMPELLO (ALICANTE) MEDIANTE LA REHABILITACIÓN DE LAS CUEVAS DEL ANTIGUO PUERTO PESQUERO
}

\author{
Beatriz Piedecausa García ${ }^{1}$ \\ Servando Chinchón Payá ${ }^{1}$ \\ Lorena González Ciller ${ }^{1}$ \\ Remedios Climent Clemades ${ }^{2}$ \\ ${ }^{1}$ Departamento de Construcciones Arquitectónicas \\ Universidad de Alicante \\ ${ }^{2}$ Asociación Museo Historia El Campello (El Campello, Alicante)
}

\section{RESUMEN}

El futuro desarrollo urbano previsto para el frente marítimo ubicado junto al antiguo puerto pesquero y actual puerto deportivo de El Campello (Alicante) se plantea con un uso terciario y comercial abusivo implicando la pérdida del paisaje existente, de la imagen de la fachada marítima y de los valores socioculturales de la zona.

Para evitar este desenlace, se plantea la recuperación de las antiguas cuevas existentes en ficho frente para uso y disfrute de sus habitantes; intervención enmarcada dentro de una propuesta de regeneración paisajística y social mediante un proyecto museístico sobre la historia arqueológica y cultural del municipio.

Con el fin de confirmar la idoneidad y viabilidad de la propuesta planteada, se hace imprescindible la caracterización geológica y constructiva de las cuevas preexistentes para así acreditar la posibilidad de recuperación de un entorno que puede llegar a reconvertirse en punto de encuentro y disfrute paisajístico comunitario.

Palabras clave: cueva, vivienda enterrada, hábitat primitivo, caracterización.

\section{ABSTRACT}

The forthcoming urban development planned for the waterfront of the ancient fishing port and modern port of El Campello (Alicante) will provoke its transformation into a commercial area, involving the loss of the existing landscape, the seafront image and the historic and cultural values in the area.

To avoid this, we propose the rehabilitation of the existing ancient caves in the waterfront for its inhabitants to use and to enjoy. This is an intervention framed into a major project to regenerate the landscape and social activities through a proposal of an archaeological museum in the municipality. 
To confirm the appropriateness and viability of the architectural proposal, it is essential to study the geological characterization and the construction system of the caves in order to prove the possibility of recovery of the natural environment to become into a meeting point and a place full of common enjoyment of the landscape.

Keywords: cave, buried dwelling, primitive habitat, characterization.

\section{Introducción}

El Campello es una población situada en la denominada Costa Blanca, a $10 \mathrm{~km}$ al norte de Alicante; de importante tradición marinera, su historia está estrechamente ligada al mar. Tanto es así que, a finales del S. XVIII, se estableció en estas tierras el primer astillero en la desembocadura del río Seco (Montnegre), convirtiéndose en la principal actividad económica de la localidad y dando lugar a una significativa industria pesquera durante el siglo XIX.

El municipio cuenta con uno de los yacimientos arqueológicos más relevantes del Mediterráneo como es la Illeta dels Banyets (Figura 1), donde se ha manifestado una antigüedad de más de 5.000 años con la existencia de vestigios de la Edad de Bronce (s. XVI-XI antes de Cristo), de la época Ibérica y del Imperio Romano (s. I-III después de Cristo).

Junto a esta zona, existe una torre levantada en 1554 denominada Torre de la Illeta (Figura 1), que forma parte de un sistema de torres vigías repartidas por la costa para proteger de los ataques de los piratas árabes y que constituye el símbolo de la ciudad visible de día y de noche. Restaurada en 1991, fue declarada entonces como Bien de Interés Cultural (BIC).
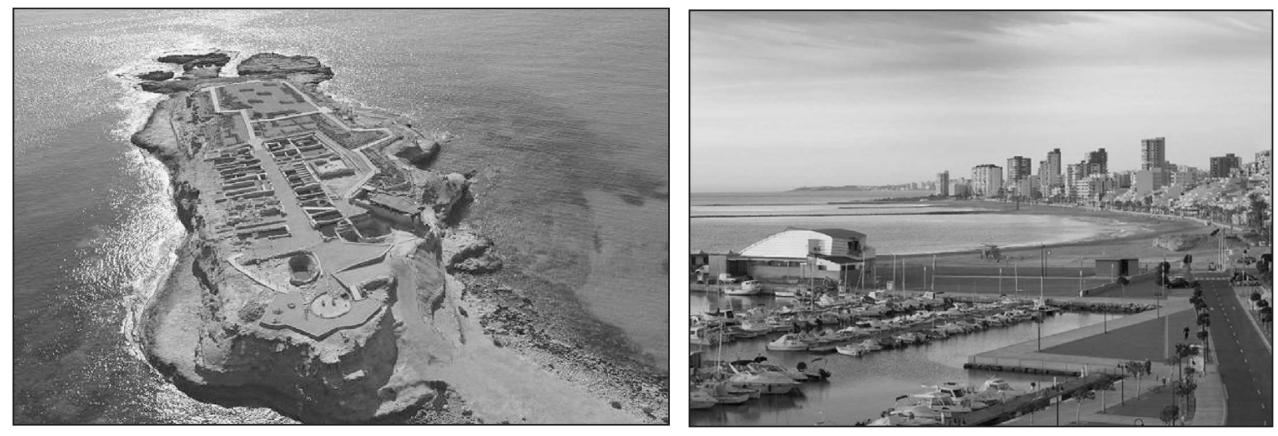

FIGURA 1. Imagen izquierda: Illeta dels Banyets. Fuente: www.elcampello.es. Imagen derecha: Puerto deportivo y puerto pesquero. Fuente: www.elcampello.es.

Descendiendo desde la citada torre hacia el nivel del mar, en un desnivel de unos $15 \mathrm{~m}$ de altura con pronunciada pendiente, se encuentran las cuevas objeto del presente estudio, ubicadas en la zona del antiguo puerto pesquero (recientemente transformado en puerto deportivo) y cuya procedencia se remonta a varios siglos atrás.

Según Gomis, J. (1999) «El origen de las Cuevas del Racó de la Illeta se pierde en la bruma de los tiempos, ya que si en el islote de Els Banys de la Reina se localizan restos de la Edad Antigua (íberos, griegos y romanos), también en las inmediaciones de estas cuevas abundan vestigios de construcciones de aquellos tiempos.»

Aún así, lo cierto es que en diversos escritos conservados se han encontrado datos de ocupación de las citadas cuevas desde hace más de cuatrocientos años. Ubicadas ante el puerto natural, en una pequeña cala protegida de los vientos de levante y de los tempora- 
les, a lo largo de su historia las cuevas han asumido diferentes utilidades. En un principio sirvieron como escondites de contrabando y a medida que la pesca proliferaba en la zona se utilizaron como almacenes, como refugio o como vivienda permanente de los pescadores o los trabajadores de la torre vigía próxima (Figura 2).

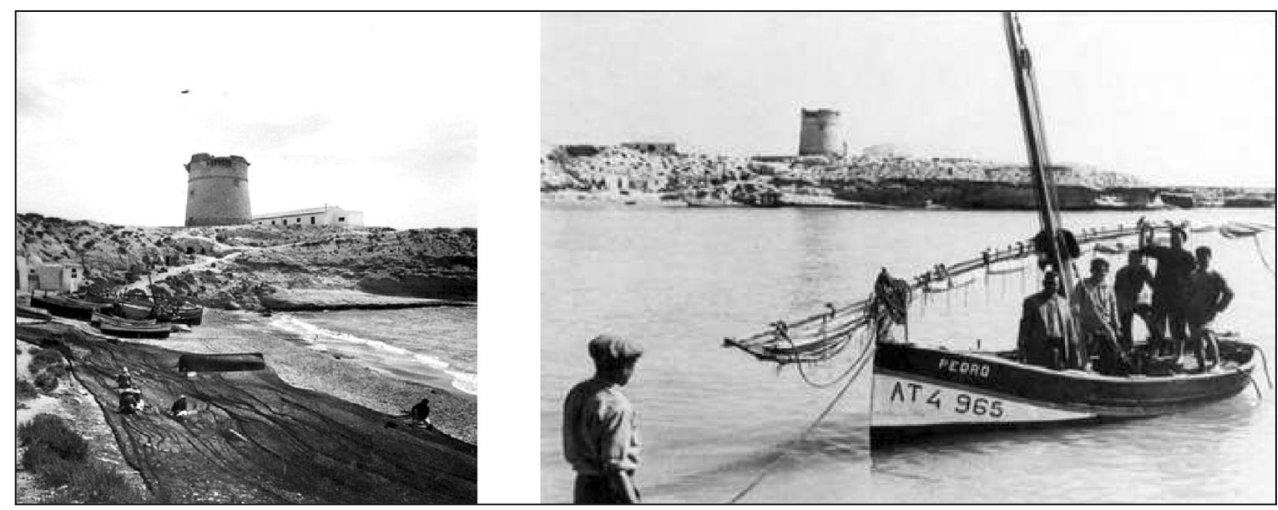

FIGURA 2. Imagen izquierda. Extendiendo las redes en el Clot de l'Illot en una imagen de mediados del siglo XX. Fuente: Olcina, M.H. (2009). Imagen derecha. Varado de barcas en el Clot de l'Illot al sur de la Illeta. Se observa un llaüt, embarcación típica de la zona que iba aparejada con vela latina. Fuente: Olcina, M.H. (2009).

Gentes procedentes del interior o de municipios costeros vecinos llegaron en busca de trabajo en la pesca y se instalaron en estas cuevas; a medida que consiguieron beneficios las abandonaron para instalarse en pequeñas viviendas construidas en los alrededores. De este modo, a fines del XIX, la zona estaba poblada por unas cuantas casitas de pescadores y esta ocupación temporal dio lugar, posteriormente, al nacimiento del Barrio de Pescadores; primero con pequeñas construcciones frente al mar, junto a las cuevas y más tarde con viviendas permanentes agrupadas en la zona de playa, al sur del puerto natural.

A este respecto, a principios del siglo XX, Rafael Altamira ofrece una visión de las formas de vida de la población de El Campello utilizando los hechos narrados en su obra El Reposo y en algunos de los Cuentos de Levante; ambos situados en El Campello aunque con nombres ficticios (Campello era nombrado como Villa-Mar). Relatos que describen las costumbres de las distintas clases sociales durante el cambio de siglo desde la experiencia directa de su autor, quien pasó muchas de sus vacaciones en esta tierra durante su infancia y también aquí se refugió en su madurez.

En las citadas obras, Altamira describe un ambiente eminentemente rural, en el que la vida diaria de campesinos y pescadores se concentraba en la obtención del sustento. En estas fechas, el desarrollo urbano de El Campello ya era considerable y las cuevas iban quedando poco a poco abandonadas, pasando a ser ocupadas por gente humilde y sin recursos.

Cuevas a las que se accedía mediante senderos en zig-zag con una importante pendiente que, según Amores, J.J. (2009), provistas de cierres de tablas servían como almacén de los enseres de pesca o como refugio permanente de los más pobres, sobre todo en los años 40, siendo incluso un refugio preventivo en caso de bombardeo durante la guerra civil.

En la actualidad, tras la remodelación urbana de la zona del puerto deportivo, de momento se ha mantenido la existencia de dichas cuevas al estar ubicadas todavía en terrenos de propiedad particular, aunque no está claro si se han respetado por su valor cultural e histórico o por dificultad de edificar en la pronunciada pendiente de la zona. 
De este modo, a pesar de que siguen conservándose, su existencia se hace apenas imperceptible desde el paseo marítimo debido a la abundancia de la vegetación circundante. Además, el mantenimiento es nulo en la mayor parte de las estancias, siendo utilizadas como refugio de indigentes o encontrándose colmatadas parcialmente con basuras.

\section{Objeto del estudio}

\subsection{Entorno geológico}

Las citadas cuevas están excavadas en materiales cuaternarios pleistocenos de origen aluvial situados sobre calcarenitas bioclásticas del Mioceno (Figura 3).

La zona se sitúa sobre un talud prácticamente vertical, en un área en la que no se descarta la posibilidad de procesos de intrusión marina y riesgo de inundaciones.

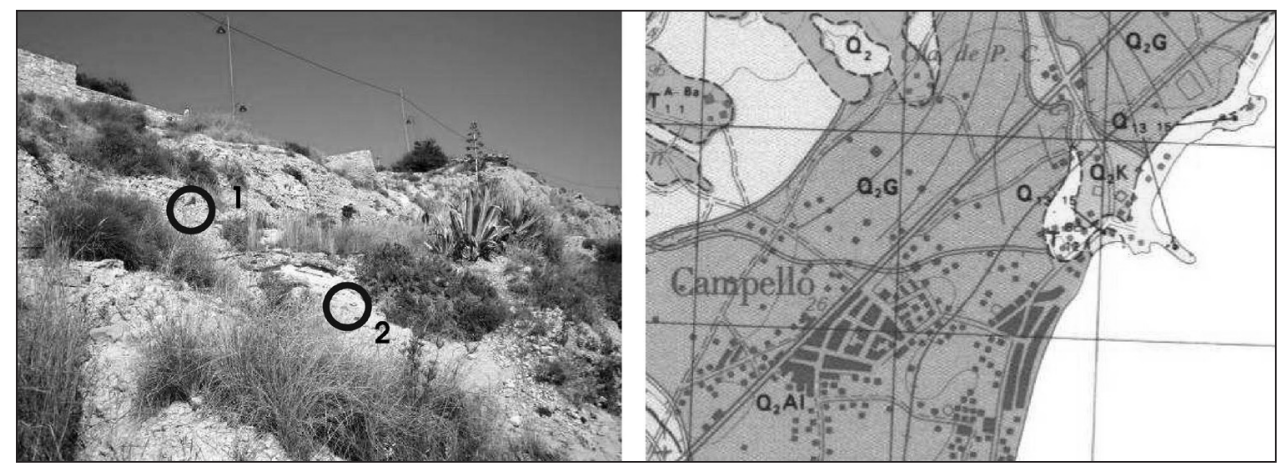

FIGURA 3. Imagen izquierda: Terreno natural del emplazamiento. Materiales cuaternarios (1) y calizas bioclásticas infrayacentes (2). Foto del autor. Imagen derecha: Mapa geológico de la zona de estudio. Las franjas de color amarillo y rosa palo, que corresponden a los materiales $\mathrm{T}_{12}$ (calcarenitas bioclásticas) y $\mathrm{Q}_{1315}$ (arenas y areniscas bioclásticas) respectivamente. Fuente: Mapa Geológico de España. Escala 1:50.000. Hoja de Alicante (872 / 29-34).

\subsection{Objetivos}

Según el Plan General del Ayuntamiento de El Campello (revisado en abril de 2010), la citada zona de las cuevas de marineros se delimita como de uso terciario, respetando únicamente un minúsculo sector próximo a la torre vigía y al yacimiento arqueológico de la Illeta dels Banyets libre frente a la urbanización masiva del resto del entorno circundante.

Tanto las estrategias de destrucción del entorno previstas por el plan para este lugar como la actual situación de abandono de estos espacios excavados, suponen una verdadera falta de consideración a la memoria histórica de la zona del antiguo puerto de pescadores de El Campello, menospreciando la presencia de las citadas cuevas y aproximando, de una manera extrema, a estos bienes culturales del municipio el uso terciario desmesurado ya existente a lo largo de toda la costa campellera.

Ante la indefensión de esta parte del patrimonio municipal, Lorena González Ciller ha planteado una interesante propuesta alternativa al planeamiento ya aprobado. Propuesta con la que se pretende revitalizar el citado entorno mediante la rehabilitación de las cuevas para nuevos usos (Figura 4) y la creación de recorridos de conexión de los diversos focos 
históricos existentes en la zona, permitiendo así el acceso a las cuevas como espacios estanciales y de actividad. Estos recorridos desembocan en un espacio museístico público que recoge la historia municipal y, además, dan apoyo a las actividades de escenificación habituales que tienen lugar en el entorno próximo como pueden ser los actos de las fiestas patronales o distintos espectáculos de música y danza. Una alternativa al plan municipal que está siendo firmemente apoyada por los representantes de la Asociación del Museo de Historia de El Campello (AMHIEC), al considerarla como una adecuada solución de recuperación del patrimonio municipal que invita al uso y disfrute del entorno marítimo.

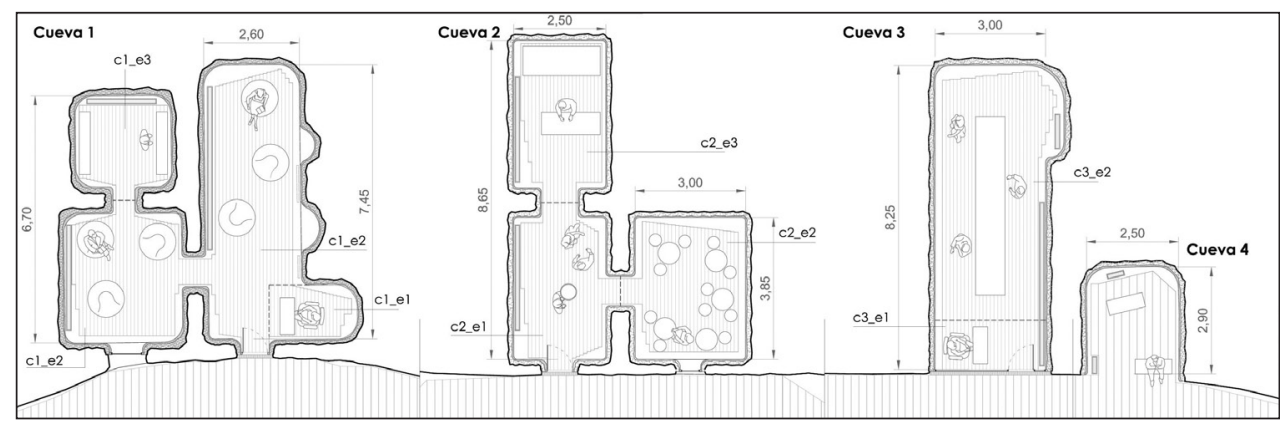

FIgURA 4. Propuesta de reutilización de las cuevas de pescadores. Aparecen nuevos programas de uso como espacio de lectura, espacio de degustación o espacio de exposiciones. Elaboración propia.

Por tanto, el objeto del presente estudio es el análisis y la caracterización de las muestras de los revestimientos en las estancias interiores y de los terrenos donde se encuentran las antiguas cuevas de los marineros de El Campello, con el fin de valorar la viabilidad de su recuperación como patrimonio histórico del municipio y proponer el cambio de su titularidad privada a titularidad municipal para su uso y disfrute de manera pública.

\subsection{Ubicación de las cuevas estudiadas}

Las citadas cuevas que se encuentran ubicadas en torno al actual puerto deportivo y antiguo puerto pesquero de El Campello (Figura 5 y Figura 6), formando un complejo de 6 habitáculos excavados en las lomas que han sido utilizados durante años como viviendas, depósitos y almacenes, tal y como se ha descrito anteriormente.

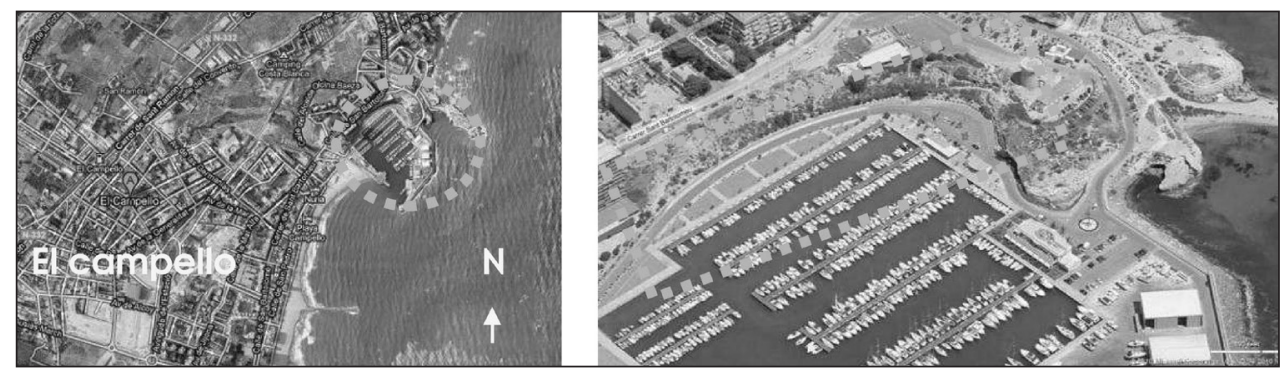

FIGURA 5. Imagen izquierda: Emplazamiento de la zona de estudio. Elaboración propia. Imagen derecha: Ubicación de la zona de estudio junto al puerto deportivo actual. Elaboración propia. 


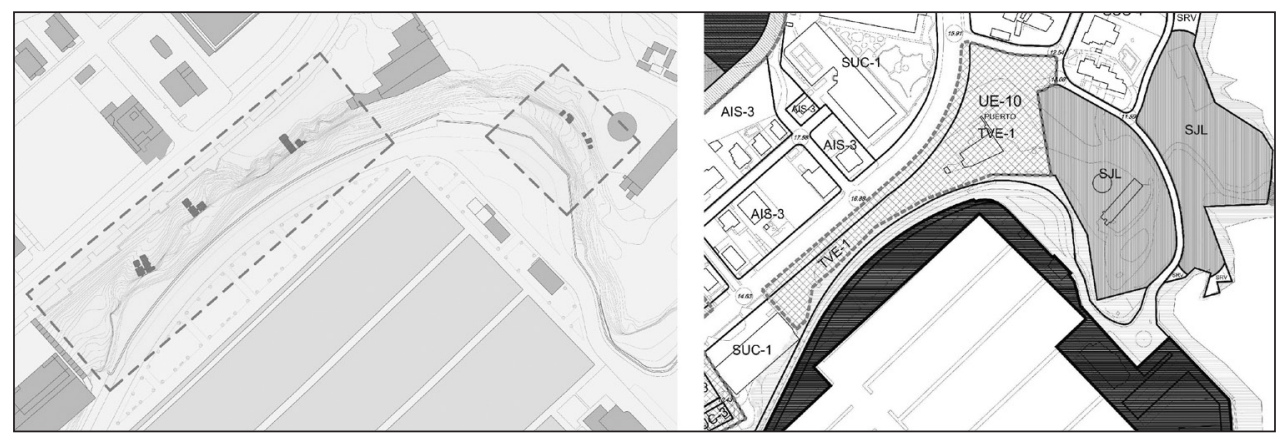

FIGURA 6. Imagen izquierda: Ubicación de las cuevas del presente estudio. Elaboración propia. Imagen derecha: Ficha de planeamiento y gestión de las actuaciones integradas previstas para el desarrollo del Plan General de El Campello. Unidad de ejecución U.E.10-Puerto. Fuente: Revisión P.G.O.U. de El Campello.

\section{Materiales y métodos}

Con el fin de caracterizar las distintas estancias, se realizó una visita donde se inspeccionaron las seis cuevas existentes. En ella, se tomaron muestras del material donde están excavadas y de la caliza bioclástica, de los revestimientos y de los compuestos neoformados que pudieran explicar posibles problemas de durabilidad. La toma se hizo en una sola campaña de muestreo y se utilizaron las medidas de seguridad y etiquetado adecuadas.

Las muestras se llevaron al laboratorio del departamento de Construcciones Arquitectónicas de la Universidad de Alicante donde se prepararon para su posterior análisis. Después de ser observadas previamente con la ayuda del microscopio óptico, se seleccionaron porciones representativas que se trituraron en un mortero de ágata hasta un tamaño inferior a 40 micras y se analizaron, mediante difracción de rayos X (DRX), en un difractómetro de polvo en los Servicios Científico-Técnicos de la Universidad de Alicante. Por otra parte, en algunos casos se guardaron también pequeños trozos de muestra para ser analizados mediante microscopía electrónica de barrido (MEB).

En la Figura 7 se adjunta un plano general de localización de las cuevas visitadas. En el apartado 2.1 se describe de forma pormenorizada el muestreo realizado en cada una de ellas.

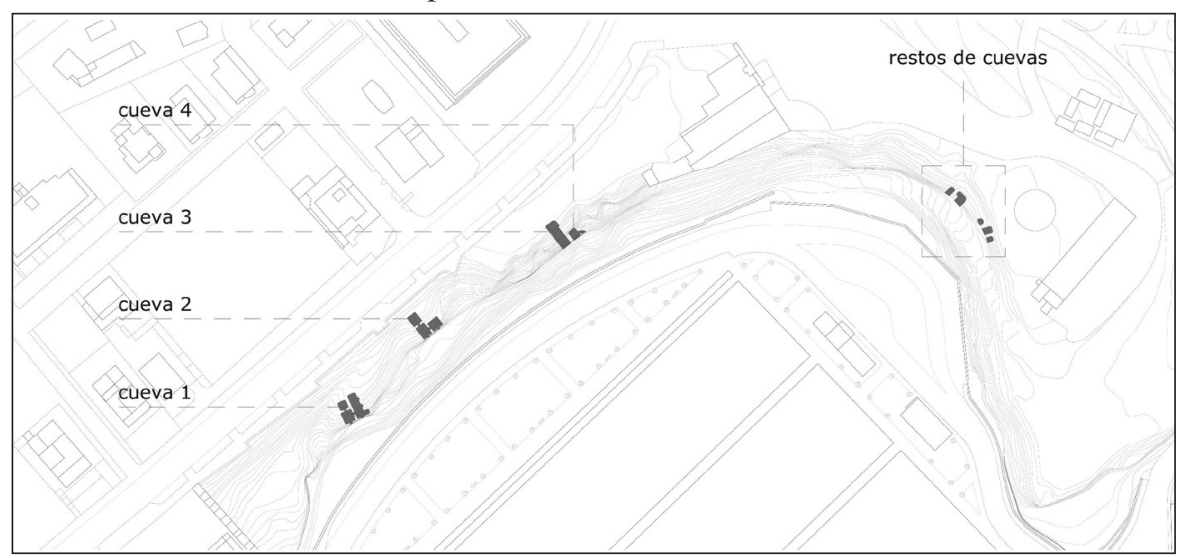

FIGURA 7. Identificación de las cuevas estudiadas. Elaboración propia. 


\subsection{Cueva 1}

La primera de las cuevas consta de tres estancias excavadas en el terreno que se encuentran comunicadas entre sí. El acceso se hace, en sentido longitudinal, por la estancia de mayores dimensiones. La primera sala se comunica transversalmente con la segunda en tamaño a través de un pequeño hueco excavado y ésta, a su vez presenta una pequeña abertura a modo de ventanal en la fachada exterior y un acceso trasero hasta la tercera de las estancias ubicada al fondo (Figura 8).

En esta cueva se tomaron un total de 3 muestras (Figura 9) correspondientes a:

- Materiales naturales de la entrada de la cueva. Muestra C1M1.

- Revestimiento artificial de la entrada de la cueva. Muestra C1M2.

- Materiales naturales de la entrada de la cueva pero de una zona distinta. Muestra C1M3.

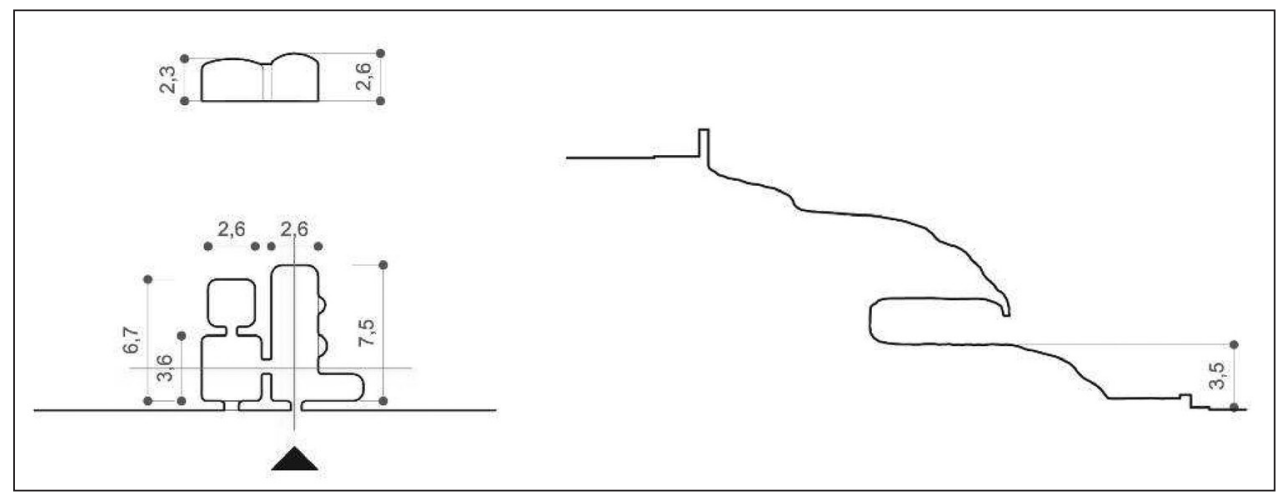

FiguRa 8. Imagen izquierda: Dimensiones en planta y sección transversal de la cueva 1. Elaboración propia. Imagen derecha: Sección longitudinal de la cueva 1. Elaboración propia.

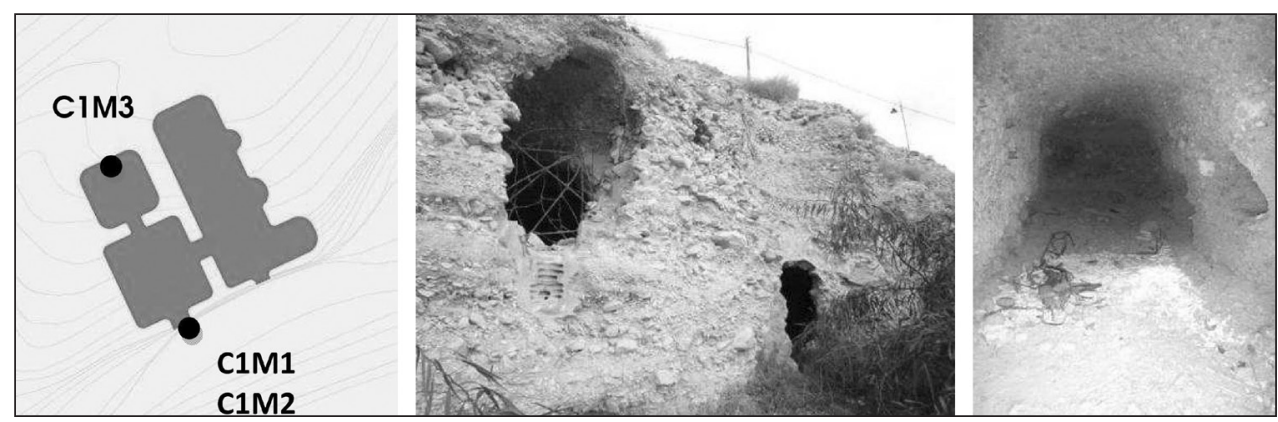

FIGURA 9. Imagen izquierda: Identificación en plano de la toma de muestras de la cueva 1. Elaboración propia. Imagen centro: Vista exterior de la cueva 1. Foto del autor. Imagen derecha: Vista interior de la cueva 1 . Foto del autor.

\subsection{Cueva 2}

La segunda de las cuevas tiene una disposición similar a la anterior. También está formada por tres estancias, pero esta vez el desarrollo hacia el interior se hace desde la sala 
de acceso. La estancia principal presenta un tamaño menor, más parecido a las otras dos salas (Figura 10).

En esta cueva también se tomaron tres muestras (Figura 11) y corresponden a:

- Revestimiento artificial blanco del interior. Muestra C2M1.

- Revestimiento artificial blanco del interior de la misma estancia pero de una zona distinta y con material terroso al dorso. Muestra C2M2.

- Materiales naturales del exterior de la cueva. Muestra C2M3.

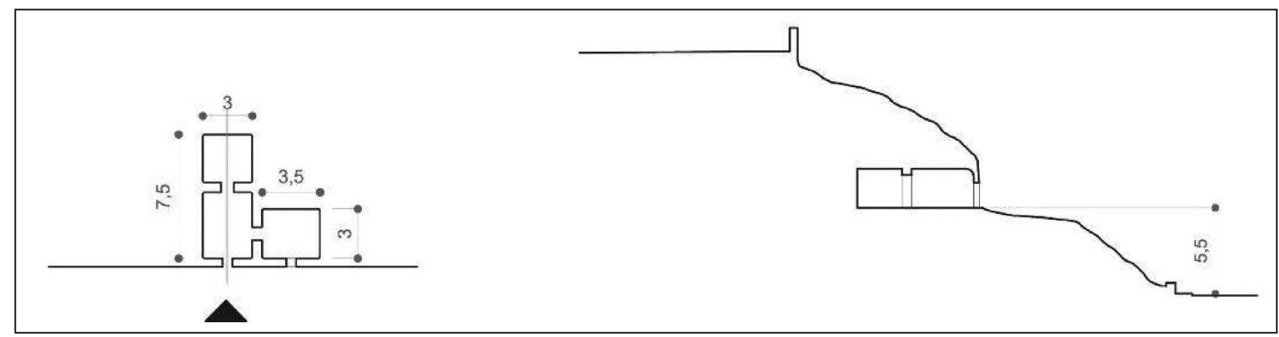

FIGURA 10. Imagen izquierda: Dimensiones en planta de la cueva 2. Elaboración propia. Imagen derecha: Sección longitudinal de la cueva 2. Elaboración propia.

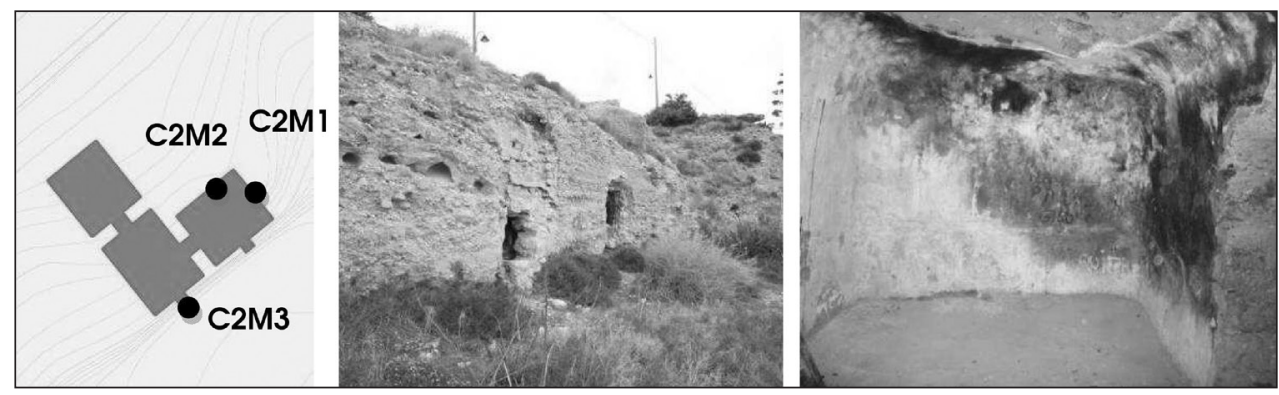

FIGURA 11. Imagen izquierda: Identificación en plano de la toma de muestras de la cueva 2. Elaboración propia. Imagen centro: Vista exterior de la cueva 2. Foto del autor. Imagen derecha: Vista interior de la cueva 2. Foto del autor.

\subsection{Cueva 3}

La tercera cueva consta únicamente de una pequeña estancia alargada y carece de revestimientos artificiales que puedan protegerla de posibles desprendimientos (Figura 12).

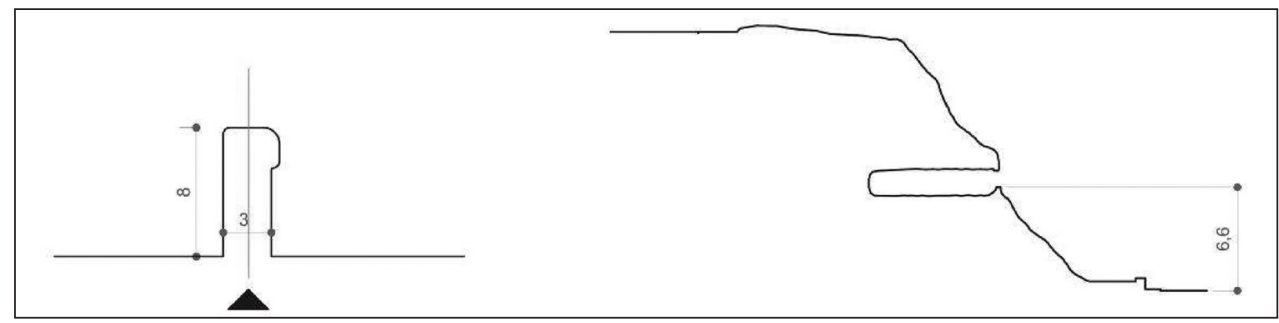

FIGURA 12. Imagen izquierda: Dimensiones en planta de la cueva 3. Elaboración propia. Imagen derecha: Sección longitudinal de la cueva 3. Elaboración propia. 
De esta cueva se tomaron dos muestras (Figura 13) correspondientes a:

- Materiales naturales del interior de la cueva. Muestra C3M1.

- Materiales naturales del interior de la cueva de la misma estancia pero de una zona distinta. Muestra C3M2.

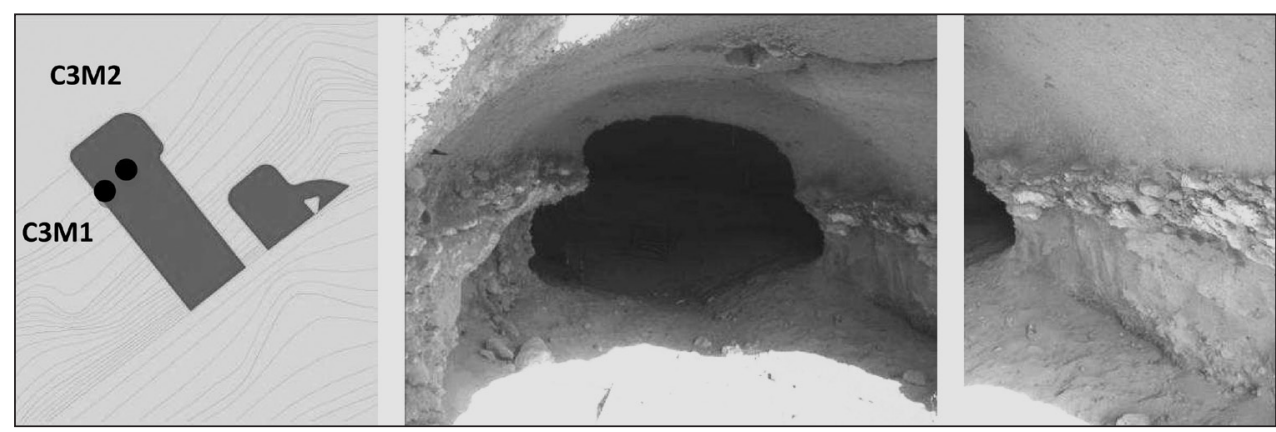

FIGURA 13. Imagen izquierda: Identificación en plano de la toma de muestras de la cueva 3. Elaboración propia. Imagen centro: Vista exterior de la cueva 3. Foto del autor. Imagen derecha: Vista lateral de la cueva 3 . Foto del autor.

\subsection{Cueva 4}

La cuarta cueva se ubica cercana a la cueva tres y es una pequeña excavación que consta de dos estancias (Figura 14). Carece de revestimientos en el interior al igual que la cueva anterior. No se tomaron muestras de la cueva 4 debido a que únicamente se encuentran los materiales naturales ya extraídos en las muestras anteriores.

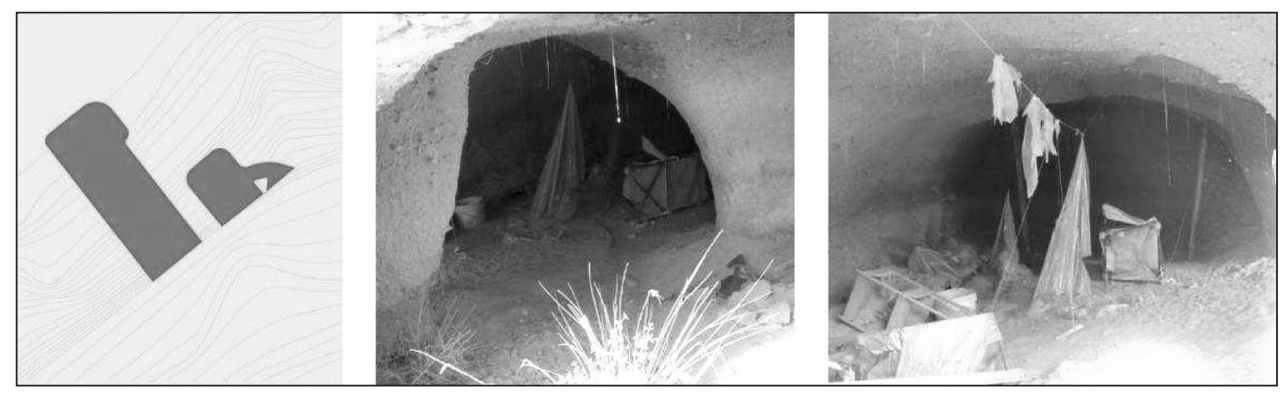

FIGURA 14. Imagen izquierda: Identificación en plano de la cueva 4. Elaboración propia. Imagen centro: Vista interior de la cueva 4. Foto del autor. Imagen derecha: Vista interior de la cueva 4. Foto del autor.

\subsection{Cuevas 5 y 6}

Estas dos últimas cuevas son oquedades semiderruidas y de pequeño tamaño. No se tomaron muestras de la cueva 5 , mientras que de la cueva 6 se tomó una muestra C6M1 (Figura 15) de los restos de un revestimiento. 

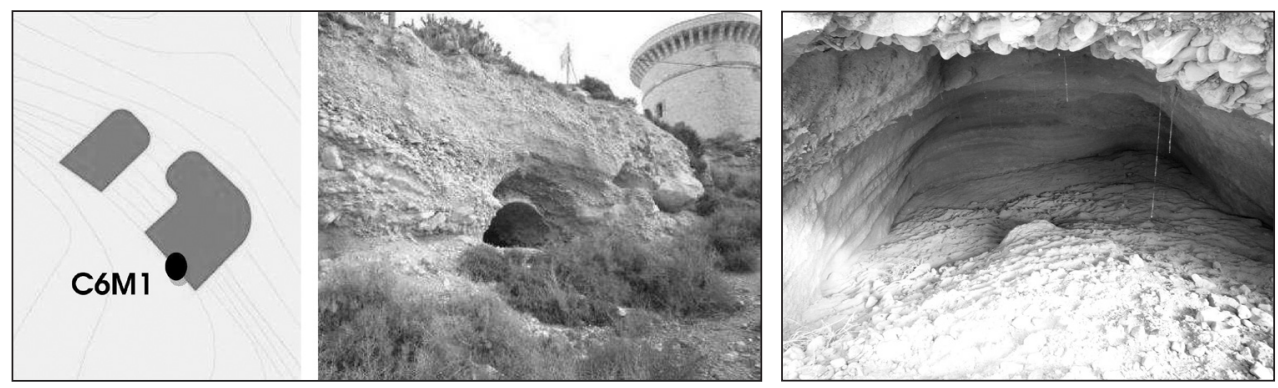

FIGURA 15. Imagen izquierda: Identificación en plano de la toma de muestras de la cueva 6. Elaboración propia. Imagen centro: Vista exterior de la cueva 6. Foto del autor. Imagen derecha: Vista interior de la cueva 6 . Foto del autor.

\subsection{Paramento PI}

Se decidió tomar una muestra correspondiente a los materiales naturales de la ladera o loma donde se encuentran excavadas las cuevas (P1M1) (Figura 16).

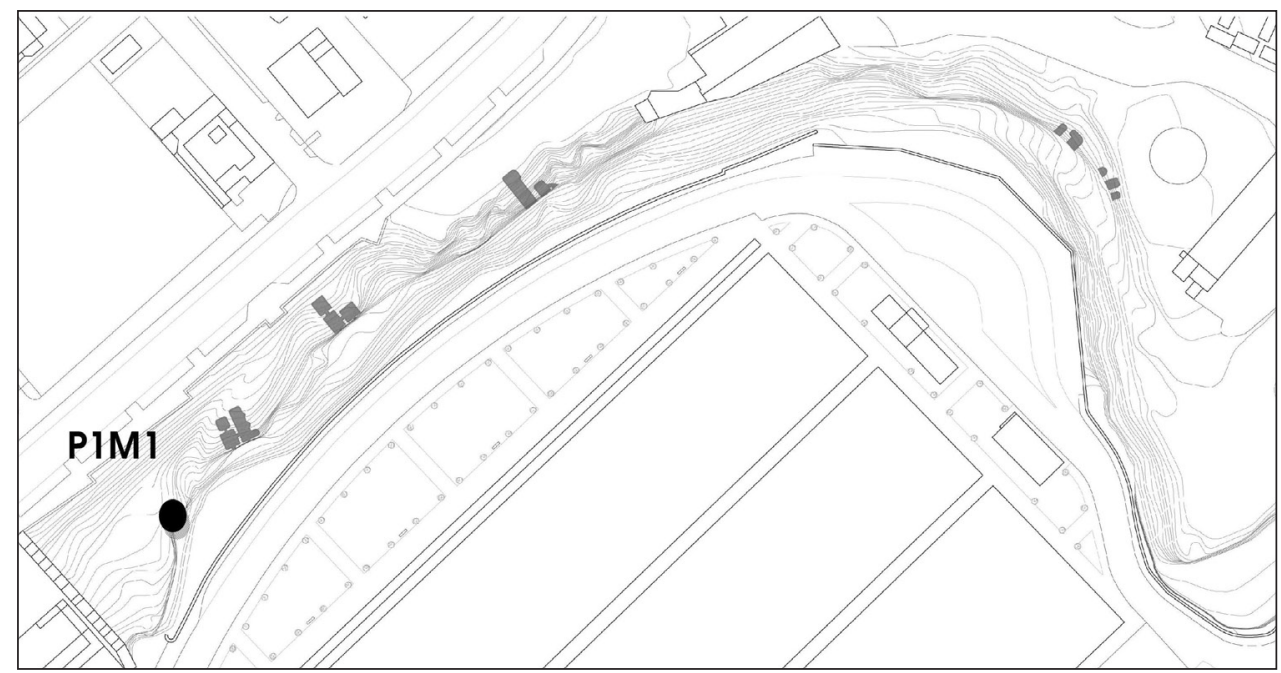

Figura 16. Identificación en plano de la toma de muestras del paramento. Elaboración propia.

\section{Resultados}

El número de muestras que se recogieron, en total 9, puede organizarse en los tres subgrupos descritos a continuación.

\subsection{Materiales naturales del entorno}

Estas muestras suponen todos los materiales naturales donde están excavadas las cuevas y son de dos tipos: muestras de cuaternario y caliza miocena. 
Las muestras de cuaternario están constituidas por cantos rodados cementados (Figura 17 izquierda). Los difractogramas correspondientes a las seis muestras de la entrada de las cuevas muestran una mineralogía similar: calcita $\mathrm{CaCO}_{3}$ y una muy pequeña cantidad de cuarzo $\mathrm{SiO}_{2}$ (Figura 17 derecha). Por tanto, los clastos y el material cementante (cuaternario) son de naturaleza caliza.

En la Figura 18 izquierda se muestra el aspecto de la caliza miocena (en la Foto 1 se observan diversos ejemplares de foraminíferos en una imagen de microscopía electrónica de barrido) y en el difractograma de la Figura 18 derecha se comprueba que se trata de una calcita magnesiana pura como muestra el espaciado reticular de la reflexión más intensa de $3,01 \AA$.
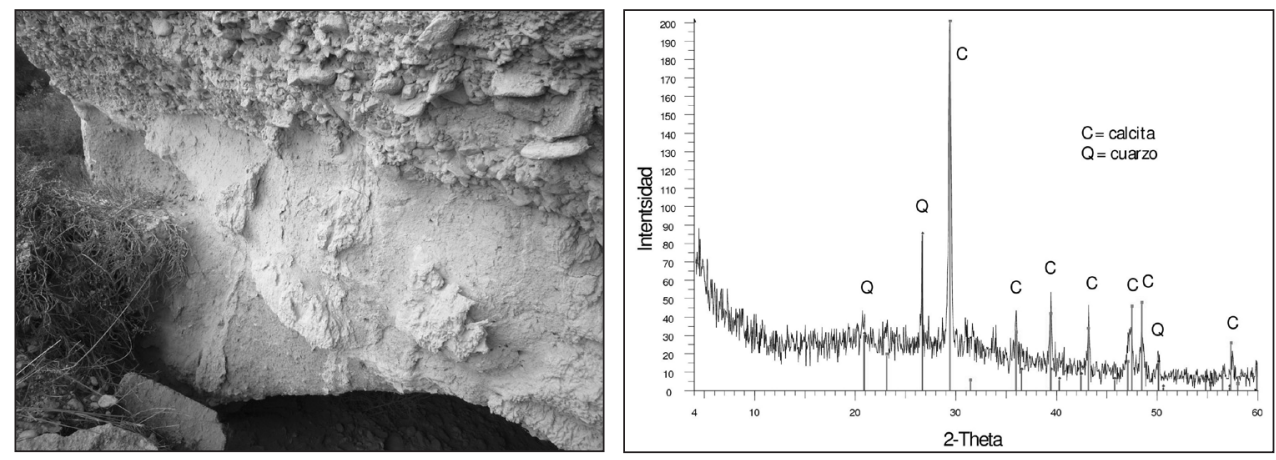

FIGURA 17. Imagen izquierda: Materiales aluviales a la entrada de la cueva. Foto del autor. Imagen derecha: Difractograma con la composición mineralógica. Elaboración propia.
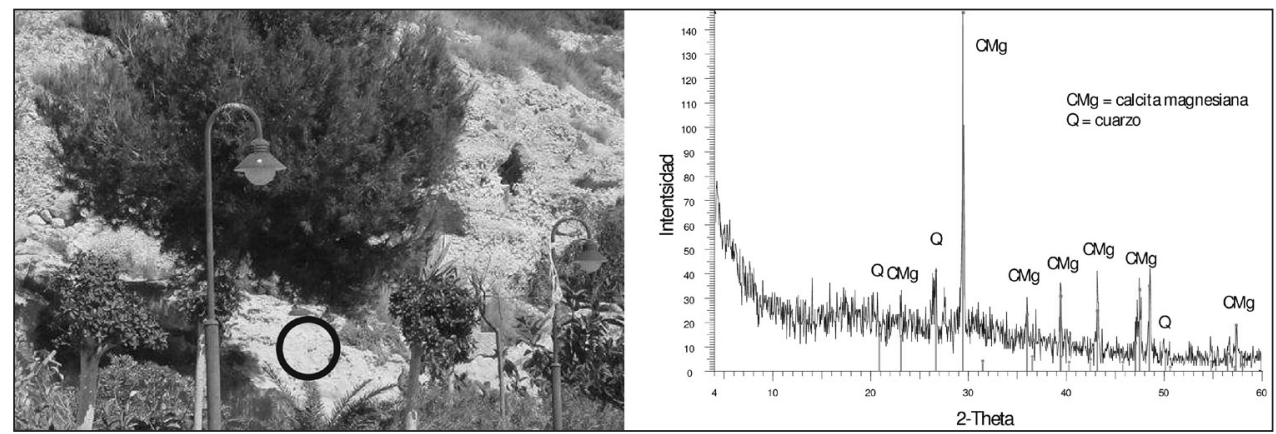

FIGURA 18. Imagen izquierda: Caliza bioclástica miocena. Foto del autor. Imagen derecha: Difractograma con la composición mineralógica. Elaboración propia.

\subsection{Revestimientos}

Debido a la dificultad que supone excavar en materiales aluviales, la entrada y el interior de las cuevas han sido revestidos con yeso $\mathrm{CaSO}_{4} \cdot 2 \mathrm{H}_{2} \mathrm{O}$ (Figura 19 izquierda y Foto 2). En un caso, concretamente en la cueva 1, el paramento de entrada está enfoscado con mortero de cemento en una intervención más reciente (Figura 19 derecha).

Es importante reseñar que en una zona de la cueva 2, en la muestra C2M1 se ha encontrado anhidrita $\mathrm{CaSO}_{4}$ en lugar de yeso (Foto 3). Eso supone que en esa parte se 
han encendido hogueras que habrían transformado el yeso en anhidrita de acuerdo con la reacción:

$$
\mathrm{CaSO}_{4} \cdot 2 \mathrm{H}_{2} \mathrm{O} \rightarrow \mathrm{CaSO}_{4}+2 \mathrm{H}_{2} \mathrm{O}
$$

La Figura 20 izquierda corresponde al difractograma del revestimiento C2M1 y la Figura 20 derecha corresponde al difractograma del revestimiento C2M2.
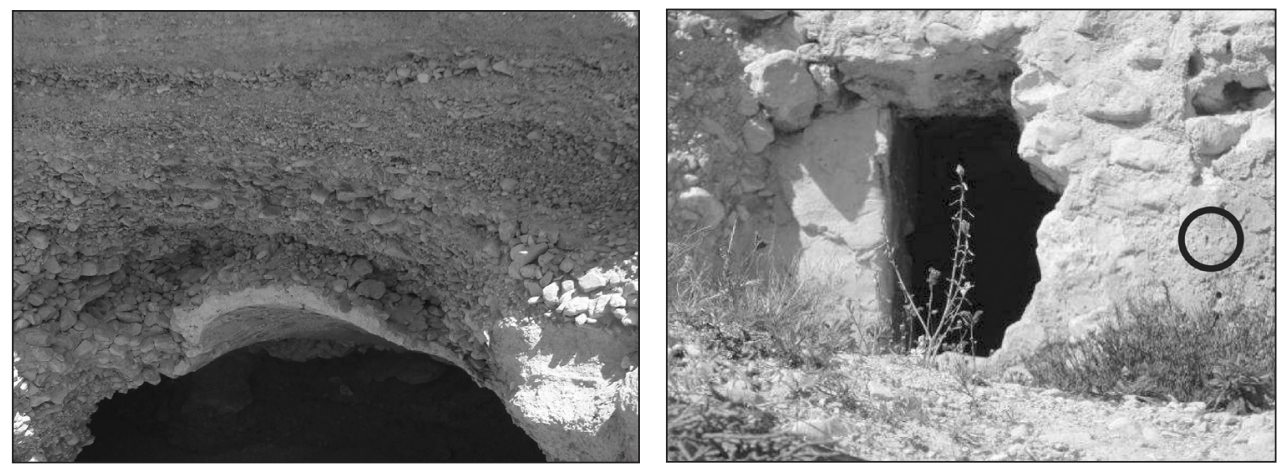

FIGURA 19. Imagen izquierda: Materiales cuaternarios revestidos de yeso. Foto del autor. Imagen derecha: Enfoscado de cemento a la entrada de la cueva 1. Foto del autor.

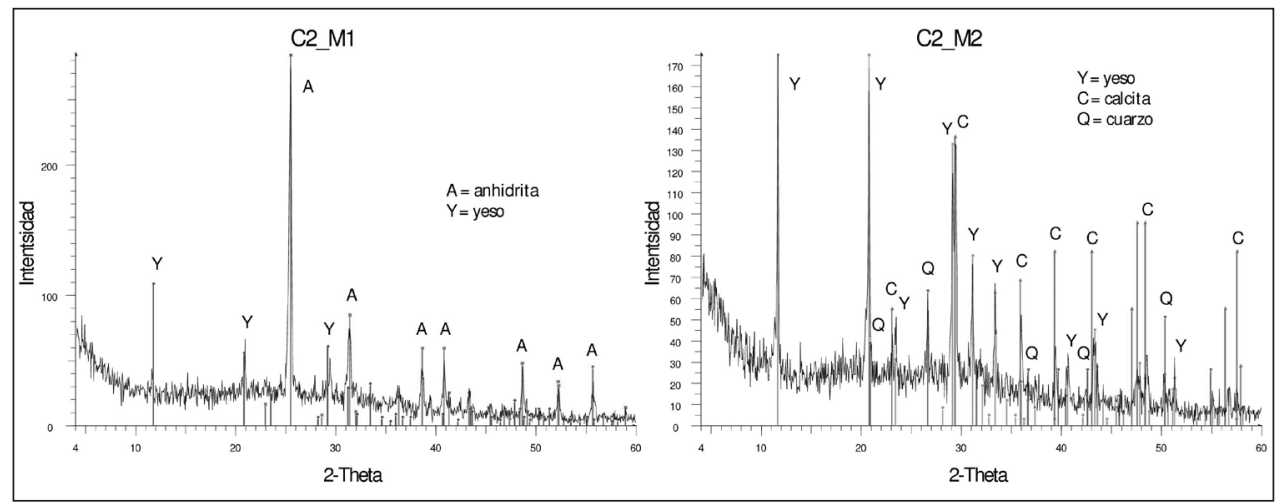

FIGURA 20. Imagen izquierda: Difractograma del revestimiento artificial blanco del interior. Elaboración propia. Imagen derecha: Difractograma del revestimiento artificial blanco de la misma estancia pero de una zona distinta y con material terroso al dorso. Elaboración propia.

\subsection{Productos de neoformación}

Como resultado del efecto del aerosol marino y en algún caso, además, como consecuencia del revestimiento de yeso, se han encontrado dentro de las cuevas sales solubles que, por su carácter expansivo, afectan a la durabilidad y la estabilidad del terreno. En la Figura 21 se muestran las reflexiones de la halita $\mathrm{NaCl}$, la mirabilita $\mathrm{NaSO}_{4} \cdot 10 \mathrm{H}_{2} \mathrm{O}$ y la thenardita $\mathrm{NaSO}_{4}$ (Fotos 4 y 5). 


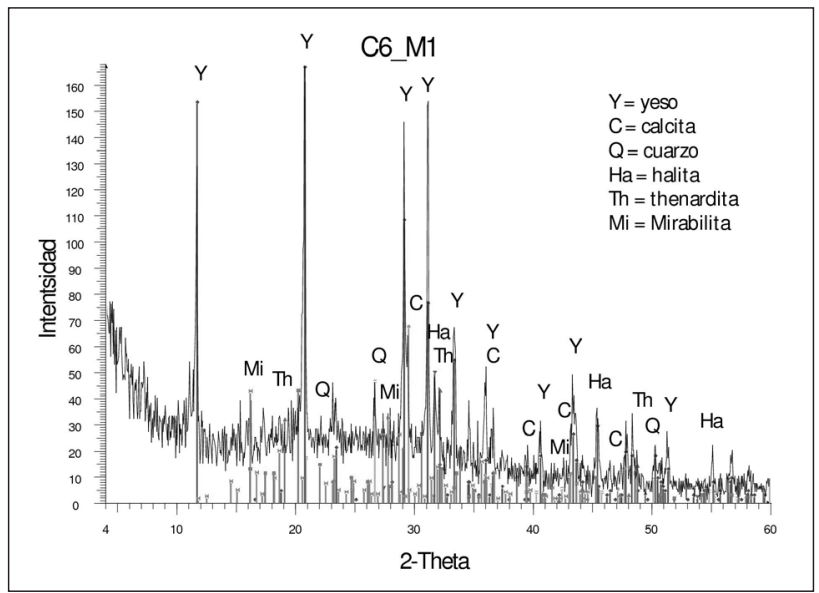

FIgURA 21. Espectro de difracción de la muestra C6M1 en el que se encuentran restos del revestimiento de yeso y productos neoformados bajo la influencia de aerosol marino. Elaboración propia.

A continuación se muestran las fotos de microscopía electrónica de barrido de las muestras analizadas, que han sido referenciadas a lo largo del texto.

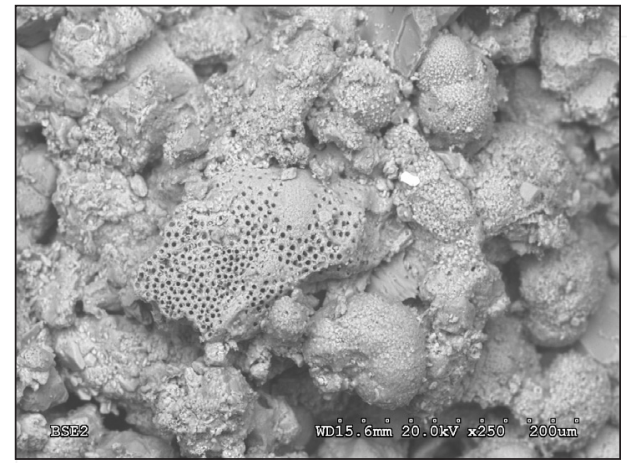

Fото 1. Diversos ejemplares de foraminíferos. Foto del autor.

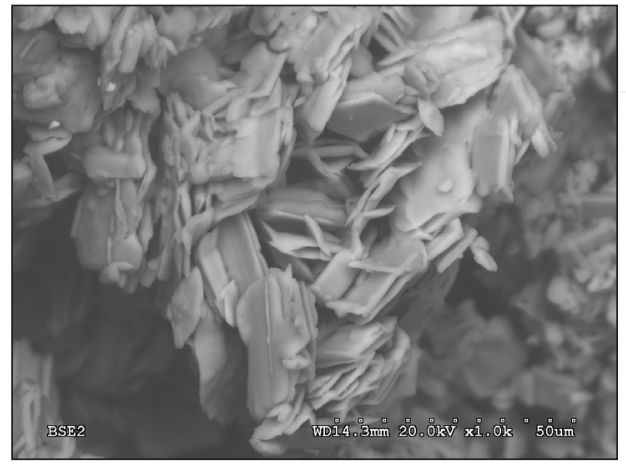

Fото 3. Anhidrita $\mathrm{CaSO}_{4}$ encontrada en la muestra C2M1. Foto del autor.

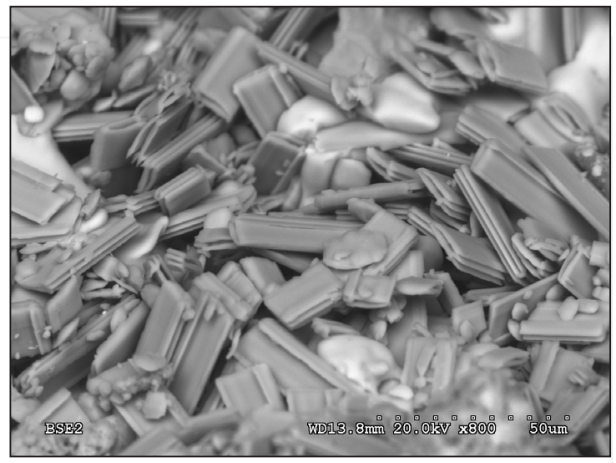

Fото 2. Yeso $\mathrm{CaSO}_{4} \cdot 2 \mathrm{H}_{2} \mathrm{O}$. Foto del autor.

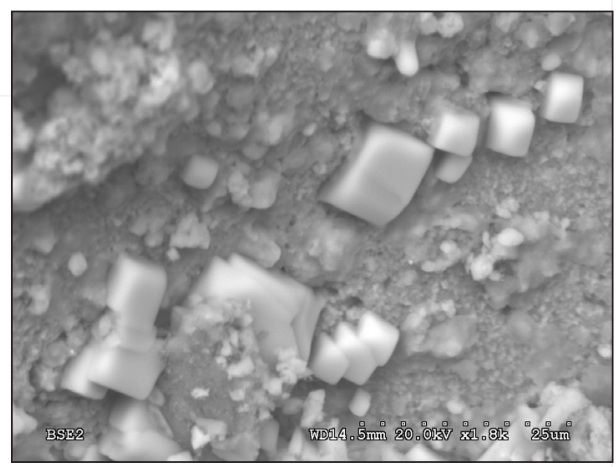

Fото 4. Halita $\mathrm{NaCl}$ encontrada en la muestra C6M1. Foto del autor. 


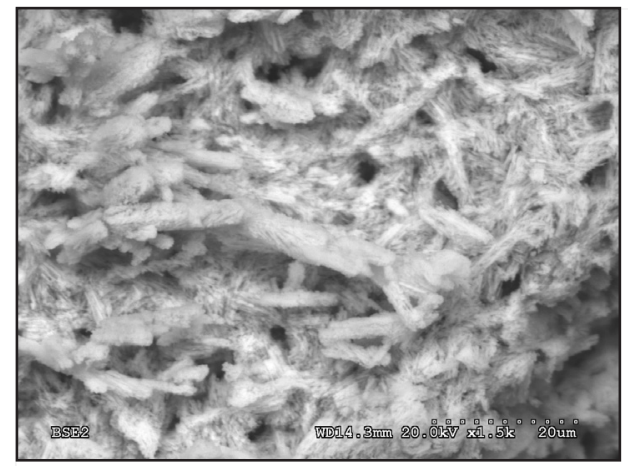

Fото 5. Mirabilita $\mathrm{NaSO}_{4} \cdot 10 \mathrm{H}_{2} \mathrm{O}$ encontrada en la muestra C6M1. Foto del autor.

\section{Discusión}

Las cuevas estudiadas están construidas sobre materiales cuaternarios o sobre calizas miocenas infrayacentes. Se trata de terrenos base fáciles de horadar y excavar, similares a los de otros lugares de la provincia de Alicante, que son susceptibles de un desarrollo arquitectónico enterrado, como ocurre en el caso de las casas-cueva de Crevillente.

La información obtenida de las muestras estudiadas, utilizando técnicas de microscopía electrónica y difracción de rayos X, indica que los materiales cuaternarios están compuestos por clastos de naturaleza predominantemente caliza cementados por calcita. La caliza miocena está formada por calcita magnesiana como es frecuente en lugares al lado del mar.

Varias cuevas están revestidas de yeso y en un caso el paramento de entrada está enfoscado con mortero de cemento portland. Especialmente el cemento, pero también el yeso, son compuestos inadecuados y responsables de la aparición de sales solubles tan frecuentes en este tipo de ambiente marítimo. Se han encontrado fases neoformadas como son los sulfatos y cloruros de sodio que pueden representar problemas de durabilidad en las construcciones.

Con el fin de adecuar las condiciones interiores de los espacios al nuevo uso propuesto sería necesario plantear un acabado interior de cal. Así, se propone una rehabilitación de las estancias excavadas mediante un material sencillo y de poco coste, acorde con las necesidades del propio terreno y que se adapta también a un ideal de bajo y fácil mantenimiento, tan necesario hoy en día en los espacios públicos urbanos.

Es importante resaltar que, si durante el proceso de rehabilitación se detectara la existencia de zonas con baja estabilidad estructural o posibilidad de desprendimientos, podría ser necesaria la incorporación de una armadura metálica de refuerzo en dichos puntos.

Por otra parte, el hecho de que las cuevas estén excavadas en un talud eminentemente vertical con la litología anteriormente descrita unido a otros factores como su situación en un área donde no se puede descartar de un modo absoluto la posibilidad de procesos de intrusión marina o el riesgo de inundaciones, hace que no sea geotécnicamente aconsejable acometer ningún tipo de edificación de gran altura, importancia o envergadura en el entorno limítrofe a las cuevas sin que estas se vean perjudicadas seriamente.

Sin embargo, al no tratarse de cargas elevadas ni de un uso agresivo ante el relieve existente, sí que parece factible su rehabilitación como espacios de apoyo con un uso liviano y respetuoso con las citadas condiciones preexistentes, tal y como se ha planteado en la propuesta de pasarelas y miradores circundantes al proyecto museístico citado (Figura 22). 
Por tanto, respecto a la viabilidad de la rehabilitación de las citadas cuevas, se puede concluir que es posible su uso como espacios de intervención sutil en el territorio, como zonas de lectura, de exposiciones o, simplemente, de descanso y disfrute de las vistas existentes. Lugares de reposo a los que acceder mediante caminos o pasarelas integradas en el paisaje, salvando el escarpado relieve de la fachada marítima y sirviendo de mirador al mar Mediterráneo.

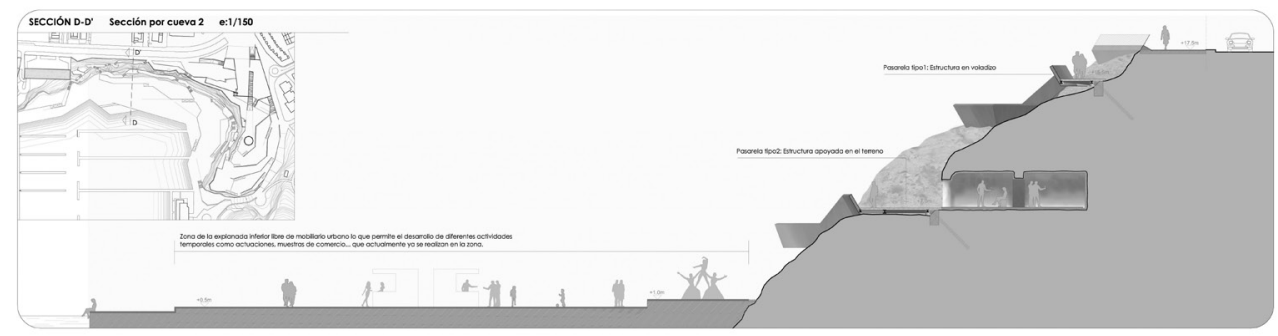

FIGURA 22. Sección de la propuesta de rehabilitación de la cueva 2. Elaboración propia.

\section{Bibliografía}

Ayuntamiento de El Campello (2011): [En línea]. <http://www.elcampello.es>. [25 de octubre de 2011]

AMORES, J.J. (2009): El Campello: huellas del aprovechamiento histórico del medio. [En línea]. Alicante, 10 de junio de 2009. <http://www.alicantevivo.org/2009/06/el-campellohuellas-del-aprovechamiento.html >. [25 de octubre de 2011]

CLIMENT, R. (1990): El Campello: mi pueblo. Graficas Mesa. Alicante.

GOMIS LLEDÓ, J. (1999): Campello entrañable. Gráficas Olmedilla. Alicante.

Instituto Geológico y Minero de España. Mapa Geológico de España. Escala 1:50.000. Hoja de Alicante (872 / 29-34).

Investigaciones Geológicas y Mineras S.A. (INGEMISA) (1991): Mapa Geocientífico de la provincia de Alicante, escala 1: 200.000. Agència de Medi Ambient y Conselleria d'Administració Pública de la Generalitat Valenciana. Valencia.

OLCINA DOMÉNECH, M. H.; MARTÍNEZ CARMONA, A.; SALA SELLÉS, F. (2009): La Illeta dels Banyets. El Campello, Alicante. Épocas Ibérica y Romana I. Historia de la investigación y síntesis de las intervenciones recientes (2000-2003). Diputación de Alicante y Museo Arqueológico Provincial de Alicante (MARQ). Alicante.

Revisión del Plan General de Ordenación Urbana de El Campello 2010. Ficha de planeamiento y gestión de las actuaciones integradas previstas para el desarrollo del Plan General de El Campello. Unidad de ejecución U.E.10: Puerto.

SANTO, J. (2003): El Campello. Municipio del siglo XXI. Editorial Everest. El Campello. 